\title{
Raphe Nuclei
}

National Cancer Institute

\section{Source}

National Cancer Institute. Raphe Nuclei. NCI Thesaurus. Code C97335.

A group of nuclei that are located in the midline of the brainstem and release serotonin. 